\title{
THE DEGREE OF PRIMITIVE SEQUENCES AND ERDŐS CONJECTURE
}

\author{
I. LAIB ${ }^{1^{*}}$ \\ ${ }^{1}$ ENSTP, Garidi Kouba, 16051, Algiers, and Laboratory of Equations with Partial Non \\ Linear Derivatives, ENS Vieux Kouba, Algiers, Algeria. \\ *Corresponding author. E-mail: laib23@yahoo.fr
}

\section{DOI: $10.20948 /$ mathmontis-2021-52-4}

Summary. A sequence A of strictly positive integers is said to be primitive if none of its term divides another. Z. Zhang proved a result, conjectured by Erdös and Zhang in 1993, on the primitive sequences whose the number of the prime factors of its terms counted with multiplicity is at most 4 . In this paper, we extend this result to the primitive sequences whose the number of the prime factors of its terms counted with multiplicity is at most 5 .

\section{INTRODUCTION}

A sequence $\mathrm{A}$ of strictly positive integers is said to be primitive if none of its elements divide another. From the sequence of prime numbers $P=\left(p_{n}\right)_{n \geq 1}$ we can construct an infinite collection of primitive sequences. According to the prime number theorem, the n-th prime number $p_{n}$ is asymptotically equal to $n \log n$; this ensures the convergence of the series

$$
f(P)=\sum_{p \in P} \frac{1}{p \log p} .
$$

A computation for $f(P)$ was obtained in [1] by Cohen as:

$$
f(P)=1.63661632335126086856965800392186367118159707613129 \ldots .
$$

Throughout this paper, we let $\Omega(a)$ denote the number of prime factors of a counted with multiplicity. For a primitive sequence $A$ the number $\max \{\Omega(a): a \in A\}$ is called the degree of $A$. It is noted $\operatorname{deg}(A)$. By convention $\operatorname{deg}(\{1\})=\operatorname{deg}(\varnothing)=0$. For any primitive sequence $A$ we pose $f(A)=\sum_{a \in A} \frac{1}{a \log a}$. We agree that $f(A)=0$ if $\operatorname{deg}(A)=0$. For any primitive sequence $A$ and any integer $m \geq 1$, we put:

$$
\begin{aligned}
A_{m} & =\left\{a \in A, \text { the prime factors of } a \text { are } \geq p_{m}\right\}, \\
A_{m}^{\prime} & =\left\{a \in A_{m}, p_{m} \mid a\right\}, \\
A_{m}^{\prime \prime} & =\left\{\frac{a}{p_{m}}: a \in A_{m}^{\prime}\right\} .
\end{aligned}
$$

Then we have $A_{i}^{\prime} \cap A_{j}^{\prime}=\emptyset$ for $\mathrm{i} \neq \mathrm{j}$ and $A=\bigcup_{m \geq 1} A_{m}^{\prime}$ is disjoint. In the case when $A$ is finit, we have $\operatorname{deg}\left(A_{m}^{\prime \prime}\right)<\operatorname{deg}(A)$. In [2], Erdös proved that the series $f(A)$ converges for any primitive sequence $A$ and in [3], Erdős asked if it is true that $f(A) \leq f(P)$ for any primitive sequence $A$. In [4], Erdős and Zhang showed that $f(A) \leq 1.84$ for any primitive sequence $A$, and in [5], Clark improved this result $f(A) \leq e^{\gamma}$ (where $\gamma$ is the Euler constant) 
in the special case when A is a primitive set of composite numbers. Several years later in [6], Lichtman and Pomerance proved that $f(A)<e^{\gamma} \cong 1.781$. Moreover, in [2], Erdös conjectured that $f(A) \leq f(P)$ for any primitive sequence $A$, then in [7,8], Zhang proved this conjecture for any primitive sequence $A$ of degree $\leq 4$ and for some special cases of primitive sequences. In [9], the auteurs simplified the proof of Zhang over the primitive sequences of degree $\leq 4$. In this note, we prove this result:

Theorem. For any primitive sequence $A$ where $\operatorname{deg}(A) \leq 5$, we have:

$$
\sum_{a \in A, a \leq n} \frac{1}{a \log a} \leq \sum_{p \in P, p \leq n} \frac{1}{p \log p} \text { for } n>1 .
$$

The proof of this result is based on the upper bound of $f\left(A_{i}^{\prime}\right)$ where $\mathrm{i} \geq 1$. We introduce the following constants, $\mathrm{K}_{0}=0, \mathrm{~K}_{1}=0.1578, \mathrm{~K}_{2}=0.4687, \mathrm{~K}_{3}=1.1971, \mathrm{~K}_{4}=2,77258, \alpha=1.11012$ and $\beta=0.0642$. We define the sequences $\left(\left(\chi_{i}(m)\right)_{m \geq 1}\right.$ as follows: $\chi_{i}(m)=1$ for $m \geq 2, j \in$ $\{1,2,3,4\}$ and $\chi_{4}(1)=1, \chi_{3}(1)=1.096, \chi_{2}(1)=1.03, \chi_{1}(1)=1.012, \chi_{0}(1)=1$.

\section{MAIN RESULTS}

We need the following lemmas.

Lemma 2.1 Let $n>1$ be an integer, put $F(n)=\log n+\log \log n-1$ then we have

$$
\begin{aligned}
& p_{n} \geq n F(n), \text { for } n \geq 2([10]) \\
& p_{n} \geq n\left(F(n)+\frac{\log \log n+2.25}{\log n}\right), \text { for } n \geq 2([10]) \\
& p_{n} \leq n(F(n)+\beta), \text { for } n \geq 7022 \\
& p_{n}>n(\log (n F(n))-\alpha), \text { for } n \geq 2 .
\end{aligned}
$$

Proof. Inequality (3) stems from inequality $p_{n} \leq n(\log n+\log \log n-0.9385)([11])$. According to (2) we have:

$$
\frac{p_{n}}{n}-\log (n F(n)) \geq-1-\log \left(1+\frac{(\log \log n-1}{\log n}\right)+\frac{\log \log n+2.25}{\log n} \text { for } n \geq 3 .
$$

Knowing that the function

$$
x \mapsto f(x)=-1-\log (1+(\log \log x-1) / \log x)+(\log \log x+2.25) / \log x
$$

is increasing on $\left[41 \times 10^{3},+\infty\right)$, then $\frac{p_{n}}{n}-\log (n F(n)) \geq f\left(41 \times 10^{3}\right) \geq-\alpha$. A computer calculation shows that, for $2 \leq n \leq 41 \times 10^{3}$ we have :

$$
\frac{p_{n}}{n}-\log (n F(n)) \geq-\alpha
$$

This completes the proof of (4). 
Lemma 2.2 For $m \geq 1$ and $j \in\{1,2,3,4\}$, we have:

$$
\sum_{i \geq m} \frac{\chi_{j}(m)}{p_{i}\left(K_{j}+\log p_{i}\right)}<\frac{\chi_{j-1}(m)}{K_{j-1}+\log p_{m}} .
$$

Proof. For $j \in\{1,2,3,4\}$, we put $N=7022, C=0.00654$,

$$
\begin{aligned}
& U_{1}=0.02348, U_{2}=0.17929, U_{3}=0.54349, U_{4}=1.30221 ; \\
& V_{1}=0, \quad V_{3}=0, \quad \text { and } V_{4}=-0.05804 .
\end{aligned}
$$

It is clear that for $m \geq N$ and $j \in\{1,2,3,4\}$ we have:

$$
\begin{aligned}
C & \geq-\log (F(m))+\log \left(1+\frac{1}{m}\right)+\log (F(m+1)+\beta) \\
C & \leq U_{j}-K_{j-1} \\
V_{j} & =\alpha-K_{j}+2 U_{j}-1 .
\end{aligned}
$$

We put

$$
h_{j}(m)=\sum_{i \geq 1} \frac{\chi_{j}(m)}{p_{i}\left(K_{j}+\log p_{i}\right)} .
$$

By (1) and (4) we have, for $m \geq N$ and $j \in\{1,2,3,4\}$,

$$
p_{i}\left(K_{j}+\log p_{i}\right)>i(\log (i F(i))-\alpha)\left(K_{j}+\log (i F(i))\right),
$$

Since $x \rightarrow \log (x F(x))$ increases for $x \geq 3$, it follows that

$$
h_{j}(m+1)<\int_{m}^{\infty} \frac{d t}{t(\log (t F(t))-\alpha)\left(\log (t F(t))+K_{j}\right)},
$$

use the change of variable $x=\log t$, we obtain:

$$
h_{j}(m+1)<\int_{\log m}^{\infty} \frac{d t}{(\mathrm{~L}(\mathrm{x})-\alpha)\left(\mathrm{L}(\mathrm{x})+K_{j}\right)} \text {, where } \mathrm{L}(\mathrm{x})=\log \left(\mathrm{e}^{\mathrm{x}} \mathrm{F}\left(\mathrm{e}^{\mathrm{x}}\right)\right) .
$$

Since, for $x>\log N$,

$$
\frac{1}{L^{\prime}(x)}<\left(1-\frac{1}{L(x)-1}\right)
$$

then

$$
h_{j}(m+1)<\int_{\log m}^{\infty} \frac{\left(1-\frac{1}{L(x)-1}\right) L^{\prime}(x) d x}{(\mathrm{~L}(\mathrm{x})-\alpha)\left(\mathrm{L}(\mathrm{x})+K_{j}\right)}
$$

by setting $y=L(x)$ and $y_{m}=L(\log m)$ we get:

$$
h_{j}(m+1)<\int_{y_{m}}^{\infty} \frac{(\mathrm{y}-2) d y}{(\mathrm{y}-1)(\mathrm{y}-\alpha)\left(\mathrm{y}+K_{j}\right)} .
$$


For $m \geq N$ and $j \in\{1,2,3,4\}$ we put:

$$
g_{j}(m)=\frac{\chi_{j-1}(m)}{K_{j-1}+\log p_{m}}
$$

then according to (3) and (5) we have:

$$
\begin{gathered}
g_{j}(m+1) \geq \frac{1}{K_{j-1}+\log ((m+1)(F(m+1)+\beta))} \\
>\frac{1}{\log (m F(m))+U_{j}}=\int_{y_{m}}^{\infty} \frac{d y}{\left(y+U_{j}\right)^{2}} .
\end{gathered}
$$

We have for $m \geq N$ and $j \in\{1,2,3,4\}$,

$$
(\mathrm{y}-2)\left(y+U_{j}\right)^{2}-(\mathrm{y}-1)(\mathrm{y}-\alpha)\left(\mathrm{y}+K_{j}\right) \leq 0 .
$$

So, for $m \geq N$ and $j \in\{1,2,3,4\}$, we have $h_{j}(m+1)<g_{j}(m+1)$ i.e.

$$
h_{j}(m)<g_{j}(m) \text { for } m>N \text {. }
$$

For $1 \leq \mathrm{m} \leq \mathrm{N}$ and by definition of $\chi_{j}(i)$, we have for $\mathrm{j} \in\{1,2,3,4\}$ a computer calculation shows that:

$$
\begin{gathered}
\sum_{i \geq m} \frac{\chi_{j}(i)}{p_{i}\left(K_{j}+\log p_{i}\right)}=\sum_{i \geq m}^{N} \frac{\chi_{j}(i)}{p_{i}\left(K_{j}+\log p_{i}\right)}+h_{j}(N+1) \\
<\sum_{i \geq m}^{N} \frac{\chi_{j}(i)}{p_{i}\left(K_{j}+\log p_{i}\right)}+\frac{1}{\log (N F(N))+U_{j}} \\
<g_{j}(m) .
\end{gathered}
$$

This completes the proof.

Lemma 2.3. Let $m \geq 1$ be fixed and let $B=B_{m}$ be primitive with $\operatorname{deg}(B) \leq 4$. For $1 \leq t \leq 5-\operatorname{deg}(B)$, we have:

$$
\begin{aligned}
& \sum_{b \in B} \frac{1}{b\left(t \log p_{m}+\log b\right)}<\frac{\chi_{t-1}(m)}{K_{t-1}+\log p_{m}}, \\
& \sum_{b \in B} \frac{1}{b\left(t \log p_{m}+\log b\right)}<\frac{1}{\log p_{m}} .
\end{aligned}
$$

Proof. For $m \geq 1$ and $1 \leq t \leq 5-\operatorname{deg}(B)$ put

$$
g_{t}(B)=\sum_{b \in B} \frac{1}{b\left(t \log p_{m}+\log b\right)} \text { where }\left(g_{t}(\varnothing)=0\right) \text {. }
$$

By induction on $\operatorname{deg}(B)$. If $\operatorname{deg}(B)=1$ and $1 \leq t \leq 4$ we have $t \log p_{m} \geq t \log 2>K_{t}$, so according to Lemma 2.2, we get: 


$$
\begin{aligned}
g_{t}(B)= & \sum_{b \in B} \frac{1}{b\left(t \log p_{m}+\log b\right)}<\sum_{i \geq m} \frac{1}{p_{i}\left(t \log p_{1}+\log p_{i}\right)} \\
& \leq \sum_{i \geq m} \frac{\chi_{t}(i)}{p_{i}\left(K_{t}+\log p_{i}\right)}<\frac{\chi_{t-1}(m)}{K_{t-1}+\log p_{m}}
\end{aligned}
$$

We assume that inequality (6) is true for $1 \leq \operatorname{deg}(B) \leq s<5$ and $1 \leq t \leq 5-$ $\operatorname{deg}(B)$ and we show that it remains true for $\operatorname{deg}(B)=s-1$. We have $B=\bigcup_{i \geq m} B_{i}{ }^{\prime}$ is dis joint, so we have:

$$
g_{t}(B)=\sum_{i \geq m} g_{t}\left(B_{i}{ }^{\prime}\right)
$$

Let $\mathrm{i} \geq m$. If $\operatorname{deg}\left(B_{i}{ }^{\prime}\right) \leq 1$ we have:

$$
g_{t}\left(B_{i}{ }^{\prime}\right)<\frac{1}{p_{i}\left(t \log p_{1}+\log p_{i}\right)}<\frac{\chi_{t}(i)}{p_{i}\left(K_{t}+\log p_{i}\right)} .
$$

If $\operatorname{deg}\left(B_{i}{ }^{\prime}\right)>1$ we have:

$$
\begin{gathered}
g_{t}\left(B_{i}{ }^{\prime}\right)=\sum_{b \in B_{i}{ }^{\prime \prime}} \frac{1}{p_{i} b\left((t+1) \log p_{i}+\log b\right)} \\
=\frac{1}{p_{i}} g_{t+1}\left(B_{i}{ }^{\prime \prime}\right),
\end{gathered}
$$

since $\operatorname{deg}\left(B_{i}{ }^{\prime \prime}\right)<s$ and $t+1 \leq 5-\operatorname{deg}\left(B_{i}{ }^{\prime \prime}\right)$ so we have:

$$
g_{t+1}\left(B_{i}{ }^{\prime \prime}\right)<\frac{\chi_{t}(i)}{K_{t}+\log p_{i}}
$$

thus

$$
g_{t}\left(B_{i}{ }^{\prime}\right)<\frac{\chi_{t}(i)}{p_{i}\left(K_{t}+\log p_{i}\right)} .
$$

So from (8), (9), and Lemma 2.2, we get:

$$
g_{t}(B)<\frac{\chi_{t-1}(m)}{K_{t-1}+\log p_{m}} .
$$

For $t=1$ we get the inequality (7), which ends the proof.

Proof of theorem Let $n$ be fixed and let $A=\{a: a \in A, a \leq n\}$ be subsequence of $\boldsymbol{A}$ where $\operatorname{deg} A \leq 5$. Put $\pi(n)=m$, the number of primes $\leq n$; then $A=\mathrm{U}_{1 \leq i \leq m} A_{i}{ }^{\prime}$ is disjoint and $f(A)=\sum_{1 \leq i \leq m} f\left(A_{i}^{\prime}\right)$. Let $1 \leq i \leq m$. If $\operatorname{deg} A_{i}{ }^{\prime} \leq 1$ then $f\left(A_{i}^{\prime}\right) \leq \frac{1}{p_{i} \log p_{i}}$ and if $\operatorname{deg} A_{i}^{\prime}>1$ then

$$
f\left(A_{i}^{\prime}\right)=\frac{1}{p_{i}} \sum_{b \in A_{i}^{\prime \prime}} \frac{1}{b\left(\log p_{i}+\log b\right)}
$$


and $\operatorname{deg} A_{i}^{\prime \prime} \leq \operatorname{deg} A_{i}^{\prime}-1 \leq 4$, so according to (7), we obtain:

$$
\sum_{b \in A_{i^{\prime \prime}}} \frac{1}{b\left(\log p_{i}+\log b\right)}<\frac{1}{\log p_{i}}
$$

therefore

$$
f\left(A_{i}^{\prime}\right) \leq \frac{1}{p_{i} \log p_{i}}
$$

Thus

$$
f(A)=\sum_{1 \leq i \leq m} f\left(A_{i}^{\prime}\right) \leq \sum_{1 \leq i \leq m} \frac{1}{p_{i} \log p_{i}} .
$$

This completes the proof.

\section{CONCLUSION}

Using a new value of the constants $\chi_{i}(m)$ will prove the theorem for greater degrees. Why not establish a recursive relationship on the degree of any sequence $A$, will prove this conjecture.

\section{REFERENCES}

[1] H. Cohen, "High precision computation of Hardy-Littlewood constants", Preprint, (1991). http://www.math.u-bordeaux1.fr/cohen/hardylw.dvi.

[2] P. Erdős, "Note on sequences of integers no one of which is divisible by any other", J.Lond. Math. Soc, 10, 126-128 (1935).

[3] P. Erdős, "Seminar at the University of Limoges", (1988).

[4] P. Erdős \& Z. Zhang, "Upper bound of $\sum 1 / a_{i} \log a_{i}$, for primitive sequences", Math.Soc, 117, 891895 (1993).

[5] D. A Clark, "An upper bound of $\sum 1 / a_{i} \log a_{i}$, for primitive sequences", Proceedings of the American Mathematical Society, 123, 363-365 (1995).

[6] J. D Lichtman, \& C. Pomerance, "The Erdös conjecture for primitive sets", Proceedings of the American Mathematical Society Ser. B, 6, 1-14 (2019).

[7] Z. Zhang, "On a conjecture of Erdős on the sum $\sum 1 / a_{i} \log a_{i}$ ", J. Number Theory, 39, 14-1(1991).

[8] Z. Zhang, "On a problem of Erdős concerning primitive sequences", Mathematics of Computation, 60(202), 827-834 (1993).

[9] I. Laib. A. Derbal. R. Mechik. and N. Rezzoug, "NOTE ON A THEOREM OF ZEHNXIAG ZHANG", Math. Montis., 50, 44-50 (2021).

[10] P. Dusart, "The kth prime is greater than $k(\ln k+\ln \ln k-1)$ for $k \geq 2$ ", Math. Comp. 68(225), 411-415 (1999).

[11] G. Robin, "Estimation de la Fonction de Tchebychef $\theta$ sur le k-ième Nombre Premier et Grendes Valeurs de la Fonction $\omega(n)$ Nombre de Diviseurs premiers de n", Acta Arith, 52, 367-389 (1983).

Received October 28, 2021 